\title{
Constructing Adequate Mental Models
}

\author{
Edward M. Pogossian \\ Institute for Informatics and Automation Problems of NAS RA \\ e-mail:epogossi@aua.am
}

\begin{abstract}
Mental systems represent realities, have varying effectiveness with respect to our goals and are processed to support utilization and gain benefits from utilities.

Classifiers induced by mental systems are effective with respect to the goals insofar as regularly provide utilities and enhance effectiveness of modeling of those utilities constructively and adequately.

In the paper we discuss ontological, constructive and systemic models of mental systems, mentals, comparable by expressiveness with algorithms and natural languages, provide arguments of their adequacy for explaining, understanding and human-computer interactions as well as convince to follow the ideas of inventors of algorithms in adequate modeling of mental behavior.

To consist functional and connectivity mental models and recalling that artificial neuron nets are systems of classifiers, we provide evidence that mentals can be reduced to systems of classifiers as well.
\end{abstract}

Keywords: Mental models, Regularization, Adequate, Constructive, Explaining, Human-computer interactions, Neuron nets.

\section{Introduction}

1.1.1. We, humans, are somewhat able to represent the causers of imprints in us and the imprints themselves by classifiers of those imprints. And since imprints can follow only certain doings of their causers (either external, internal, or both), the classifiers represent the causers representing, in fact, the doings of the causers.

1.1.2. We process classifiers to preserve or provide our utilities.

We enhance effectiveness of that process, particularly, enhancing the quality of classifiers as well as uniting the efforts of members of communities for the utilities by communicatives (cms) of the classifiers, i.e., by IDs of classifiers or classified samples, as, for example, we communicate now by IDs, English words, in this paper.

1.1.3. Classifying the causers of imprints and imprints themselves as realities and their totality as our universe we assume that effectiveness of preservation of our identity is to the extent to which we, particularly, adequately represent the universe by constructive classifiers and effectively process them.

In other words, as comprehensively and adequately classifiers cover the diversity of realities as powerful we become. 
For example, each of us enhances his effectiveness in the universe growing up his language skills. Then, communities, say speaking English with more than 300 thousand of highly constructive classifiers, are incomparably powerful than tribes with languages in a few thousand words representing mainly not constructive classifiers.

And whether the first assertion of the Bible "...It was the Word in the Beginning and the Word is the God" doesn't remind that the classifiers and their cms in languages, i.e., the words, either acquired from genomes or from communities are one of fundamentals of our power...

1.2.1.In the diversity of classifiers we identify ourselves as originated and formed by communities, then as a type of cellular realities, cellulars, which, in turn, identify as an intersection of a type of not entropic, negentropic, realities [6], negs, with a type of durables, refers, i.e., realities able to preserve the identity of caused in them imprints. Consequently, humans and representations of humans, say by their doings, are, at least, dependent while mainly predetermined by fundamentals of communities, cellulars and negs.

1.2.2.Particularly, mental doings of humans are essentially predetermined by genomes and cultures of their communities implying communality of members of communities not only in innate means of representing realities by sensors and classifiers, in types of processing of those representations for variety of utilities including enhancement of effectiveness of themselves but also, in general, in commonality of particular lines of reasoning, counting, expressing them in languages, etc.

That is why the novelty of mental doings of humans, usually, is in enlightening lines of cause effect reasoning between already known communal utilities and representations of realities. And only occasionally that novelty is in discovering of new utilities or case effect reasoning why those novelties become so sound in communities up to becoming granted by Nobel Prizes.

1.2.3. Thus, reflecting the above assumptions to this research we classify it as an attempt to enlighten then generalize already known and successfully applied expertise of mental modeling by the founders of algorithms, namely, the expertise of transition from classifiers of computability to their models, algorithms.

And, apparently, in that modeling we cannot but have to heavily relay on the fundamentals of communities, cellulars and negs stated, particularly, by the following assumptions.

1.3.1.1.

Ad1. Durables are realities that in contrast with others ,temporals, have somewhat, kernel of durables (Kd), that can be properly identified in the time. .

Ad2. Refers are durables with kernels including the imprints of their causers.

Ad3. Refers able to identify classes of imprints are classifying refers.

1.3.1.2.

An1. All negs are classifying refers and do in the universe to preserve certain roots which include regular energy supply for their doings and an ability to stay classifying refers..

An2.The existence of natural negentropics different from cellulars, remains open yet while some types of artificial ones humans can already construct.

An3.While existence of types of durables seems tractable the origin of negs and cellulars (even though as unicells) remains a mystery yet.

1.3.2.

Ac1. Cellulars do to reproduce themselves, do to benefit from utilities, i.e., realities favorable for the roots, to avoid their damagers, to utilize realities uncertain yet with respect to (wrt) the roots as well as to challenge already gained utilities and, possibly, roots themselves.

Ac2.Cellulars represent realities via doings of those realities.

Ac3. The vast majority of doings of cellulars are predetermined by genomes and cultures of their communities. 
Ac4. Cellulars gain effective doings, at least, by a chance search in the space of diversely replicated doings of their cells, and, possibly, by regular cognition of regularities of the universe.

Ac5. Cellulars have sensors, or classifiers outputting identified imprints for certain inputs.

Ac6. Mental doings of cellulars are doings over imprints of certain realities, i.e., mental doers (mdoers) and systems of mdoers (mss), aimed to support doings of cellulars by their mss representing and processing.

Ac7. Mss $m$ of members $x$ of communities $C$ of cellulars have IDs (mID) and comprise certain nets $\mathrm{xN}$.

Mss $\mathrm{m}$ corresponding to mIDs, or the meanings of mIDsof $\mathrm{x}$, are connectivity subnets of the nets $\mathrm{xN}$ rooted in those mIDs, and can be activated internally or externally by their mIDs or by samples $\mathrm{r}$ of already classified by m realities.

Subsets of those mIDs and realities r communalized in C comprise communicatives (cms) of C.

1.3.3. Commenting on the assumptions and, first of all, the negentropicity of humans let's recall that entropics, following Schrödinger [6], comprise the vast majority of realities. They inevitably lose their energy, and therefore, any sign of durability.

In opposite to entropics, negentropics, negs, comprise only a small island of realities and are able to preserve certain durables, root realities, or roots in space and time, and the premise necessary to preserve the roots is their ability in regular gaining energy from others.

Roots for realities $\mathrm{r}$, we assume, are any given, usually not explained yet realities, possibly constituents or doings of $r$, that are preserved for $r$ regularly and with first priority wrt others.

Apparently, roots of negs necessarily include doings for gaining energy and ones to preserve that ability.

1.3.4. Roots of cellulars include, at least, their genomes, doings for periodic diversified reproduction of genomes and doings for preserving realities induced as auxiliary to the roots.

1.3.4.1. Mental doings are baking other doings including themselves, are either genomic or gained in the lifetime while gained mainly by acquisition from the cultures of communities.

1.3.4.2. Mdoers do over outputs of sensors and mdoers to elaborate instructions for the effectors. While they can be represented as classifiers, particularly, relationships, rules, regularities or their compositions, algorithms, we argue that they are reducible to classifiers of n-tuples of identified, nominated realities.

1.3.4.3. Mss compose mdoers to represent, particularly, systemic classifiers, say Factories, Computers, Chess Positions.

1.3.5.1. Classifiers of roots and utilities are identified as root and induced goals why cellulars can be classified as goal oriented realities.

Attributes, we assume, include classifiers of constituents of compound utilities and realities with uncertain yet utilities.

Apparently, realities can be partially ordered by degrees of their utilities wrt the roots, thus, to induce corresponding ordering for the goals and attributes.

1.3.5.2. Mss as well as their constituent mdoers can be processed for a variety of goals, particularly, to learn new utilities and to enhance effectiveness of mss.

1.4.1. While the fundamentals of mss and their processing can be found for all cellulars the highest of them are unique only for humans that can be stated, particularly, by the following assumptions.

Ah1. Doings of members of human communities are mainly equal implied by equality of 99\% of genomes of all humans and commonality of cultures of communities of their being.

Ah2. Humans adapt to the universe mainly by cognition and development of mental doings.

Ah3. Humans accumulate, reproduce effective doings then transfer them in space and time not only by genomes but also by the records of the patters of those doings that are essentially depersonalized and estranged from particular members of their communities. 
Ah4. Meanings m of mIDs of $\mathrm{x} @ \mathrm{C}$ are connectivity subnets of nets $\mathrm{xN}$ rooted in mIDs can be scaled by their effectiveness wrt utilities of $\mathrm{x}$, say constructiveness of adequate modeling of $\mathrm{m}$, and wrt explanations of $\mathrm{m}$ in $\mathrm{C}$, say completeness of $\mathrm{m}$ wrt intensions of $\mathrm{x}$ and expectations in $\mathrm{C}$.

Ah5.Mental doings classified by psychologists and psychiatrists including classifying, learning, prognosticating, communicating can be equally represented by adequate constructive models of mss and their nets.

1.4.2. Cognizers are, we assume, a type of mss while cognition includes doings in acquisition, accumulation as well as revelation, discovery of mss, particularly, by learning of new utilities or enhancement of effectiveness of the existing ones.

1.4.3. All over governing of mss including their cognition, activation and processing is realized, we assume, by controllers that, it is not excluded, can be the causers of our awareness or consciousness and be mss as well.

Particularly, controllers govern communication between the members of communities in explaining and understanding mss of each other.

1.4.4. Being realties we can classify and explain ourselves as well.

For example, controllers explain mss "Humans" of the author by resolving it into this, ongoing text, namely, corresponding English words to IDs of constituents of the mss.

In general, that resolution can start from any constituents of the target mss while their IDs can be chained causally, logically or in a variety of other modes and be detailed depending, particularly, onthe goals of the author.

1.4.5.Mainly equal doings of members of communities $\mathrm{C}$ mean, particularly, that

- $\quad$ the same any what cause equal imprints for any x,y @C , thus, $\mathrm{x}$ realities are equal to y realities what implies the universe UC equal for all members of $\mathrm{C}$,

- mental doings including classifying, learning, teaching, inference, prognostication are equal in $\mathrm{C}$ what let members of $\mathrm{C}$ to communicate doings of each other for effective collaborations.

1.5.1. A mighty way of enhancement of effectiveness of mss, and thus, cognizers, is the regularization of classifiers induced by mdoers and mss [47].

Namely, classifiers $\mathrm{Cl}$ of members $\mathrm{x}$ of communities $\mathrm{C}$ are regularized in $\mathrm{C}$ if accompanied by ontological in $\mathrm{C}$ methods, instructions allowing $\mathrm{x}$ regularly provide positive samples of inputs of $\mathrm{Cl}$ as well as let the members of $\mathrm{C}$ to do the same by communicating with $\mathrm{x}$.

In constructive regularization those samples can be provided deterministically and without any involvement of cellulars while, otherwise, can be grown up from a priory given prototypes like cells or crystals, be the products of services to humans or machines.

1.5.2. Regularly provided positives $\mathrm{r}$ of classifiers $\mathrm{Cl}$ and $\mathrm{Cl}$ themselves are interpreted as models of classifiers $\mathrm{Cl}^{\prime}$ if $\mathrm{r}$ are classified as positives of $\mathrm{Cl}^{`}$ andCl are interpreted as adequate models of Cl' if positives $\mathrm{r}$ meet certain additional requirements focused for positives of $\mathrm{Cl}$.

For example, algorithms are adequate models of deterministic methods if, following Church, to any method by certain instructions equal algorithms can be corresponded [30 ].

1.5.3. Interpreting the aims of algorithms to enhance the effectiveness of classifiers of deterministic methods we expand them to other classifiers focusing the mental ones and state the following:

S1. Algorithms are modeling and constructively regularize deterministic methods.

S2. OO Languages are constructively regularized and strongly expand algorithms.

S3. Mentals are constructively regularized and strongly expand OOL.

S.4.For languages $\mathrm{L}$ of communities $\mathrm{C}$ allowing the members $\mathrm{x}$ of $\mathrm{C}$ to communicate, i.e., to explain and understand mss of each other expressed in L, communication algorithms LC can be 
constructed letting computers communicate mental models Mns of mssMs of $\mathrm{C}$ equally wrt the members of $\mathrm{C}$ if $\mathrm{Mns}$ and Ms are equal to each other.

1.6. At present adequacy of mss is questioned functionally and connectively.

Functional questioning examines the equality of performances of mss and models of mss of any origin.

In contrast, in connectivity modeling it is required that the units of the models mss have to be adequate models of the units of nerves systems, neurons, and, particularly, looking for adequacy of mss with artificial nets of neurons, $A N N$.

Examining primarily functional adequacy of mentals and recalling ANN are systems of classifiers we provide an evidence that mentals can be reduced to systems of classifiers as well , thus, stating that

S5. Mentlals can consist of functional and connectivity mental models.

1.7. In what follows, first, we continue to develop constructive models of mss, mentals introduced in [47], to argue later that they are modeling mss adequately.

Then, refine systemic classifiers and constructive regularization of classifiers followed by overview of some ad hoc regularized classifiers.

We question the ways of proving that mentals can be adequate constructive models of mss and suggest to examine equality of performances of particular mss with corresponded them mentals as well as question the consistency of performances of structural models of connectivity neuron nets, for example, artificial neuron nets, with purely functional models of mss, mentals.

Ideally, equality of performances of mss and mentals have to be proven for all mss as well as Church thesis had to be examined for all deterministic methods.

Realistically, we focus the proof of equality of performances of mentals and some inevitable in cognition mss, including communications, explaining, understanding [47], some types of learning , acquisition and search of mss [42, 43,50], .

1.7. Our models are based on and try to fuse findings of many outstanding researchers.

We refer to some of their publications [1-35] to learn them in depth as well as refer to some works [36]-[42], which can add to understanding of our ideas and their approbations [43]-[50].

\section{Systemic vs. Do Classifiers}

2.1. Doers, in general, are, we assume, realities having in- out- put parts and for available inrealities, i.e., for realities, and more, for somewhat, at the input parts, either elaborate certain output realities or stay passive.

In- out- realities comprise their in- out-domains, or in- out-doms.

Indomswrt outputs are split into classes of equality, thus, absence of outputs corresponds to the class (?) of uncertain inrealities.

2.2. Doers are do-classifiers $\mathrm{Cl}$ if indoms are split into two classes $+\mathrm{Cl}$ and ?Cl; otherwise they are corresponders, cors.

Apparently, identifiers of do-classifiers $\mathrm{Cl}$ by themselves are sufficient to indicate their classes of equality, i.e., the positives $+\mathrm{Cl}$, while classes of cors can be indicated by pairing those identifiers with the corresponding outputs.

2.3.Realities $\mathrm{I}\{\mathrm{i}\}$ are identifiers, IDs, of realities $\mathrm{R}\{\mathrm{r}\}$ and $\mathrm{Z}\{\mathrm{z}\}$ wrt $\mathrm{Z}$ if

- to any r,z the unique IDs i(r), i(z) correspond,

- to any r,z certain classifiers are linked allowing by IDs i(r), i(z) to recall corresponding r, z,

- any $\mathrm{r}$ can address to any $\mathrm{z}$ for recalling any $\mathrm{r}, \mathrm{z}$.

Identified realities of given $\mathrm{R}, \mathrm{Z}$ paired with their IDs are named nominals wrt $\mathrm{Z}$.

2.4. Classifiers of $n$-tuples of nominals are $n$-place relationships shortly named rels for $n=2$.

Rels (a,b) can be depended or not on the orders of their arguments. 
2.5.1. Systems $H$ over nominals Nls containing rels Rls, i.e., Rls $<$ Nls, or systems $H$ over Nls/Rls, include Nls and if H' are systems of $\mathrm{H}$ then systems of any subset of H' linked to each other by rels of Rls and nominated by IDs consistent with nominations of Nls are systems of $\mathrm{H}$ as well. 2.5.2. Systems can be external and internal, particularly be mss, texts , Markov algorithms, etc. 2.5.3.The totality $\mathrm{H}$ of systems over Nls/Rls comprise $\mathrm{sNl} / \mathrm{Rls}$ nets where nodes $\mathrm{a}$, b are IDs corresponding to systems of $\mathrm{H}$, the edges ( $\mathrm{a}, \mathrm{b}$ ) correspond to rels between the systems represented by nodes a,b , signed, "colored " by IDs of those rels and oriented from b to a if correspond to rels(a,b).

$\mathrm{Nls} / \mathrm{Rls}$ nets are, in fact, colored and oriented nets where nodes $a$ depend on nodes $b$ if rels (a,b) correspond to the edges linking a and $b$.

Assuming Nls are 1st layer systems of Nls/Rls nets, the systems of $n+1$ - layers are formed as systems over nominals of $n$-th layers and relsRls.

2.6.1. Do classifiers $\mathrm{Cl}$ are defined as a type of doers that for realities at the input parts either elaborate certain outputs or stay passive, thus, splitting indoms into two classes +Cland?Cl.

We assume that not only doers but any systems, particularly mss, h induce certain systemic classifiers hsCl with positives $+\mathrm{hsCl}$ s determined as follows.

2.6.2. Realities $r, r^{\prime}$ are (fuzzy) equal with respect to doins $d$ if $d$ is applied to r,r' outputs (fuzzy) the same otids. In other words, d analyzing r, r' by their embedded regs doesn't find any (fuzzy) distinction between $r$ and $r$ '.

Due to equality of realities, as a rule, they can be incomplete, approximate or fuzzy later on in refining equality we skip to name the option of their fuzziness.

2.6.3. Doers are equal if for any inputs their outputs coincide.

For constructiveness of that requirement, we assume that indoms of doers are made finite by some criteria.

Thus, doers are equal if their performances, i.e., the pairs input/output, for inputs of their indoms coincide.

2.6.4. Systems G,G' are equal if certain doers d determine that decompositions of G,G'untill terminal doins are isomorphic wrt equality of corresponded to each other doins while those doins are linked by the equal rels.

2.7.Realities $r$ match to systems $h$ if certain doers $d$ can reveal in $r$ equal to h systems $h$ '.

Thus, pairs (mentals h, d) determine systemic classifiers hsCl with positives +hsCl.

\section{Regularized and Modeled Classifiers}

3.1. Classifiers in their min mode identify some realities while if regularized can regularly provide samples of their indoms. In turn, regularized classifiers can be models or adequate models of each other either constructive or not, as it follows.

3.2.1.Classifiers $\mathrm{Cl}$ of members $\mathrm{x}$ of communities $\mathrm{C}, \mathrm{x} € \mathrm{C}$, are regularized if $\mathrm{x}$ can accompany $\mathrm{Cl}$, first, by methods Clrgz letting $\mathrm{x}$ provide positives of $+\mathrm{Cl}$ regularly, and second, $\mathrm{x}$ by ontological in $\mathrm{C}$ communicatives Clcms can explain Clrgz to any y $€ \mathrm{C}$ why y can provide positives of $+\mathrm{Cl}$.

Regularized are, for example, classifiers of produced goods, grown up domestic plants and animals, services and skills provided hand to hand.

Scientists $\mathrm{x}$ discovered some realities a and classified them by regularized classifiers $\mathrm{Cl}$ can by Clrgz provide samples of a and by Clcms successfully explain to others how to do the same.

3.2.2. ClassifiersCl are fuzzy regularized if they are regularized but to the extent that outputs of Clrgz only fuzzy match to $+\mathrm{Cl}$ and the fuzziness can range up to not matching to $+\mathrm{Cl}$. 
3.3.1. Let's recall that positives of $+\mathrm{Cl}$ are realities of indoms of do classifiers doCl and are systems of doers for systemic classifiers sCl.

Particularly, if mss h are some do classifiers doCl positives matching $+\mathrm{hsCl}$ will be $\mathrm{doCl}$ themselves while positives of $+\mathrm{doCl}$ will be some realities of indoms of $+\mathrm{doCl}$.

3.3.2. Corollary1. Regularized mss h are equal to hrgz methods wrt out realities of hrgz, i.e., if realities rare reproducible by hrgz then they match $h$.

Apparently, regularized mss h necessarily have to include systemic classifiers hsCL either explicitly or implicitly.

Statements on mss, as a rule, alsohave transparent fuzzy interpretations, which can often be skipped later.

3.4.1.The samples of classified realities $\mathrm{r}$ can be constructed deterministically and totally independent of cellulars in constructive regularization, conrgz, or can be regularized not constructively, particularly be provided not deterministically, be grown up from a priory given realities like cells or crystals, be a product of services to humans or machines.

For example, mss Goods (Gds) representing producible in the frame of some civilization goods, saymss Computers (Cps) since are reproducible from totally not cellular realities.

Conrgz are mss Algorithms (Ags), represented either as Turing Machines, Post Productions, Markov algorithms or Recursive Functions that can be specified to be assembled from not cellular units by mathematicians or programmers, and even more, they are enumerable.

Mss Wheat, Domestic Animals are fuzzy regularized and grown up while mss Services of cellulars, say Treatments by Doctors, are fuzzy regularized and are inseparable from cellulars.

Assuming a numerical scale for fuzziness of regularization its variable $f$ might be zero for Cps, Gds and Ags, range from zero for mss Deterministic Methods (DM) to $1 / 2$ for the Heuristics, and have $\mathrm{f}=1$ for mss Conscious, Emotions, Passions questioned yet to be regularized.

3.4.2. Humans tend to regularized classifiers $\mathrm{Cl}^{`}$ for the advantage not only passively classify but actively provide positives of classifiers.

Constructive regularization let, in addition, exempt positives from being cellulars, thus, expanding leverages to amplify target doings. Recall, for example, transition from riding by horses to cars or trains.

3.5.1. Regularized mss $h$ are modeling mss $h *$ if out realities of hrgz match $h *$.

Regularized mss $h$ are adequately modeling mss $h^{*}$ if they are modeling $h^{*}$ and for any realities $r^{*}$ matching $h *$ hrgz can produce realities $r$ equal to $r^{*}$.

Apparently, regularized mss $h$ are adequately modeling themselves.

3.5.2. By Church DM can be adequately modeled by Ags, i.e., to any DM equal Ags can be corresponded.

Recalling Corollary1, it can be stated the

Corollary2. Regularized mss h are equal to certain algorithms wrt out realities matching $\mathrm{h}$.

3.5.3. Church thesis, we assume, can be expanded for fuzzy and not deterministic algorithms and methods as well.

Namely, DM can be expanded to Methods (Mds) and Ags to fAgs adding to Ags, say, probabilistic, distributed and heuristic methods / algorithms.

Corollary3. Regularized mss h (fuzzy or not) are equal to certain algorithms (fuzzy or not) wrth realities matching h. 


\section{Extending Constructive Mental Models}

4.1.1. Algorithms are a type of regularized mss adequately modeling computations as well as systems and their doings that humans can code by numeric ones equally representing the originals.

For example, we are modeling chess adequately since we can code constituents of chess and rels between them by numbers having numeric relationships between each other equally representing the relationships between original constituents of the game.

While OO languages expand numeric rels of algorithms by adding attributing/have, parenting/be and do rels they are far to represent a variety of rels we indicate by natural languages (NL).

For example, NL rels include ones such as love, sincerity, passion between humans without any idea to be ever represented numerically. Then, models of the essentials of NL, say UNL [21,2], indicate, at least, 44 basic rels of NL while there is no evidence that all of them can be represented by equal numeric ones.

4.1.2. Concluding that acceptable constructively regularized OO models for mss NL are very questionable

we are going to expand $\mathrm{OO}$ languages to systems, mentals, that

-first, are exempted from the requirement to have only numeric input IDs provided by humans, i.e., capable along numeric input IDs to operate with ones provided by the given sensors analogous with neuron nets, and

-second, let to process any relationships we identify in natural languages.

We believe that mentals can approach the adequate constructive modeling, at least, of the kernel of NL and in the next chapters we will argue the reasons for that belief.

4.2.1. Doers of type of classifiers are sensors if inrealities are not necessarily pre-classified, of type of cors are effectors if inrealities are necessarily classified while are controllers if both inout- realities are necessarily classified.

4.2.2. Controllers Cns, are assumed, can assign IDs to given mdoers aimed to control their processing and in- out- interactions with realities.

4.2.3.In identification of realities $\mathrm{R}, \mathrm{Z}$ realities $\mathrm{Z}$ can be interpreted as sets of Cns controlling in certain ways realities of $\mathrm{R}$ analogical to servers of "star" types controlling networks of computers or, seemingly, analogical to unicellular controllers.

4.3. Nominals wrt Cns where realities of R are outputs of doers, particularly, sensors, controllers or effectors, are named otids while sets of otids of doers $d$ are the alphabets of $d$.

And sets of otids comprised from only some representatives of alphabets A1,A2, ..,An of doers $\mathrm{d} 1, \mathrm{~d} 2, \ldots, \mathrm{dn}$ are words in $\mathrm{A} 1, \mathrm{~A} 2, \ldots, \mathrm{An}$.

4.4.1.For given controllers Cns, effectors Efs and sensors Sns nominated wrt to Cns, or ces, doers $D$ over IDs of ces, or cesdoins $D$, are doers $\mathrm{D}$ nominated wrt to Cns while indoms of D, Efs, Cns are words in alphabets of the outputs of D, Cns, Sns.

4.4.2. Bundles of otids of Sns,Cns and cesdoins at time $t$ are $t$ prints comprised into certain stores Pns and nominated wrt to Cns.

4.4.3.Basic ces nominals, or cesbns, include cesdoins D united with Cns, Efs,Sns,Pns nominated wrt Cns.

4. 4.4. Systems of ces bnominals, or scesbns, are systems over Nls, Rls where Nls are cesbns.

The totalities of scesbns comprise cesnets Nts.

4.5.1. While scesbns can range from the sets of disjoined to the totally connected to each other systems we refine mental systems, mss, as those of scesbns that are connectivity subnets $G$ of cesNts rooted in the nodes $a$ of Nts. 
Namely, total connectivity scesbns $G$ rooted at nodes a (or total a connectivity scesbns G) of cesNts are connectivity subnets of Nts rooted in a.

And a rooted scesbns $G$ ' of total connectivity a scesbns G, or a mentals, or, generally, mentals, are $a$ rooted connectivity subsystems of $\mathrm{G}$. Then, $a 1, a 2 \ldots$, an aspects of $G$ ' are a1,a2,.., an rooted connectivity subsystems of G'.

Apparently, connectivity a scesbns are $a$ mentals and nodes a1,a2,.., an by themselves can be the aspects of G'.

The totalities of mentals of cesNts comprise ces thesauruses cesTh.

4.5.2.Decompositions of 1st depth, or 1st decompositions, of a mentals $G$ having nodes $a$ at some layer $k$ of cesNts are a1,a2,.., an rooted mentals of all subsystems $G 1, G 2, \ldots, G n$ of $G$ with nodes $\mathrm{a} 1, \mathrm{a} 2, \ldots$, an of $\mathrm{k}-1$ layers of Nts connected to a.

And if $\mathrm{G} 1, . ., \mathrm{Gn}$ are $\mathrm{i}$-th decomposition of $\mathrm{G}$ then $i-1$ th decomposition of $G$ will be the union of 1st decompositions of G1,..,Gn.

Apparently, the terminal decompositions of $\mathrm{G}$ will be comprised from bnominals of Nts.

The unions of i-th decompositions of $\mathrm{G}$ for $\mathrm{i}=1, \ldots, \mathrm{k}-1$ comprise total decompositions of $\mathrm{G}$.

4.5.3. Analogously can be defined j-th abstractions and total abstractions of a mentals $G$ in the way that 1 st abstractions of a mentals $G$ with nodes a at some layer $k$ of ces nets Nts could be $\mathrm{a} 1, \mathrm{a} 2, .$. ,an rooted mentals $\mathrm{G} 1, \mathrm{G} 2, \ldots, \mathrm{G}$ of all subsystems of $\mathrm{G}$ with a1,a2,..,an at the $\mathrm{k}+1$ layers of Nts and connected to a, etc.

4.6.1.Thesauruses cesTh are assumed to be stored by analogy with storing libraries, say in Java.

Namely, nodes of cesnets are stored with IDs of the nodes, the classifiers of IDs of the nodes, IDs of rels of nodes a with nodes $b$ along with IDs of those $b$.

Nodes $a$ corresponded to ces abstracts $\mathrm{d}$, in addition, contain either the decision makers of $\mathrm{d}$ or the references to them.

4.6.2. Apparently, nodes corresponded to ces abstracts of cesnets or in cesTh will coincide with abstract classes of Java in the case when their rels with other nodes of cmnets are restricted by "attributed", "parented" and "done by" ones.

4.6.3. Started from identifying common for communities $C$ doers, mdoers and msystems, so far, we have specified doins, scesbns, cesnets and cesTh thesauruses as well as total connectivity scesbns, their connectivity subsystems, mentals, and the aspects of mentals representing, we assume, mss and the aspects of mss.

We question whether mentals can be adequate constructive models for mss and for that aim refine constructive modeling and adequacy as it follows.

4.7.1. The following types of scesbns can equally correspond to algorithms, say in Markov or other equal modes.

Equal to rules by Markov are types of doins, regularities, or regs, corresponding certain otids to only some selected words of indoms.

Algorithms are scesbns comprised from regs by rels similar to ones comprising rules into algorithms by Markov [29,30].

Scesbns algorithms, in fact, deepen the definition of ones by Markov detailing the origin of the rules. Namely, if Markov algorithms are defined starting from the given basic alphabets the scesbns ones assume certain sensors providing those basic alphabets.

4.7.2. Scesbns of the types of "abstract classes", referring, say, to ones in Java, are systems of algorithms, or methods, in rels of the types: "attributed", "parented" and "done by", with other abstract classes.

Abstracts expand abstract classes by allowing arbitrary Rlsrels with other abstracts.

Finally, packages of abstracts and their libraries are mimicking the ones in Java.

4.8.1.Markov algorithms, apparently, are systems of rules, regs of the types of "realities y follows realities $x$ ”, “ $y$ is function of $x$ ", " $x$ cause $y$ ”, etc. 
Recalling that logical functions and predicates can be represented by each other we can state that the above

Interpretations of Markov rules can be represented as corresponding time, case -effect, dependency rels, thus, conclude that mentals as well as ANN, in general, can be represented as systems of classifiers.

4.8.2. Addressing to the consistency of functional and connectivity mental models and recalling the diversity of current functional mental models in contrast with only classifying ANN ones as well as encouraging reducibility of mentals and ANN to systems of classifiers we find reasonable to state the question of looking for assembles of neurons in ANN providing mental doings equal to ones by mentals.

\section{Questioning Adequacy of Mentals}

5.1.1. Justification of mentals as adequate models of mss can be done by analogy with justification of algorithms as adequate models of computability by Church.

Namely, the adequacy of mentals for several mss have to be proven, then, a hypothesis $h$ are declared on adequacy of mentals to any mss that are examined empirically for mss until $\mathrm{h}$ would be refitted by some mss or another not equal to mentals alternative models of mss will be discovered.

5.1.2.Ideally, that justification means that for the original problem human-universe (HU) for systemic classifier sClm of any mss m of any $\mathrm{x} @ \mathrm{C}$ solving HU it is possible to provide mentals m' with classifier sClm' equal to sClm.

Realistically, since adequacy of mentals can be examined for finite number of mss only it is worth to examine, first of all, for certain key mss. As such key mss we select meta mss, i.e., ones doing over mss, then, ones acknowledged by psychologists, psychotherapists as nucleus in identifying the norms of being healthy humans.

5.2.1.The next barrier in justification of the adequacy of mentals is the incredibility of $\mathrm{HU}$ problem in examining equality of mentals to target mss.

Ideally, for proving adequacy of mentals m' for target mss $m$ we had to confirm equality of $m$ and $m$ ' for any type of their relevant processing for any tasks of HU problem, which is unrealistic.

To overcome that barrier we follow the views that HU problem can be approximated by game models [52]. Then, analogously with [27] we find that combinatorial games with known hierarchies of utilities and solutions in spaces of possible strategies in game trees can with a proper adequacy represent the HU problem.

Thus, we are narrowing HU to the Solvers of Reproducible Game Trees (RGT) problems, a class of combinatorial problems with only a few following requirements to belong to:

- there are (a) interacting actors ( players, competitors, etc.) performing (b) identified types of actions in the (c) specified moments of time and (d) specified types of situations,

- there are identified benefits for each of the actors,

- the situations the actors act in and transformed after the actions can be specified by certain rules, regularities.

5.2.2. The arguments of proper adequacy of RGT Solvers to HU include the following ones.

First, we find out that games with known hierarchies of utilities and solutions in spaces of possible strategies in game trees can properly represent HU problem.

Then, RGT represent combinatorial environments that, generally, cover enormous not solved yet problems [27] in contrast to ones well represented by classical continuity and parameterizations based mathematics. 
RGT is a spacious class of unsolved problems including many security and competition problems. Specifically, these are network Intrusion Protection (IP), Management in oligopoly competitions and Chess-like combinatorial problems as well as many other security problems such as Computer Terrorism Countermeasures, Disaster Forecast and Prevention, Information Security, etc. [43].

5.2.3. Along with acceptable adequacy RGTsoftware,atpresent, provides a preferable research environmentletting look for proper scientific assertions. Those preferences include the following ones.

1.Urgent and spacious RGT combating and competition problems are reducible to the standard kernel problem $\mathrm{K}$ of the class and we do focus chess as the $\mathrm{K}$. 2. K- methodology multiplies the achievements for particular problems of the SSRGT class.

3. Distributed development of K-methodology is possible.

4. K-centric methodology enhances the effectiveness of RGT Solvers providing answers to the urgent RGT questions including the following ones:

4.1. measurement of the effectiveness of Solvers,

4.2. analysis and typifying combating knowledge,

4.3. construction of knowledge based Solvers,

4.4 acquisition in a regular way RGT expert knowledge and enhancing the effectiveness of Solvers.

5. The validity of K- methodology was proved for certain RGT problems including - Chess,- Network Intrusion Protection - Navy Defense from Attacks, Management, Marketing and others.

6. The shell of RGT Solvers is developed to provide user friendly Java environment for managing any RGT problem [ 40.44-46,50].

5.3.1. At the time a variety of mss have proper algorithmic or OO models.

Let's recall some of models to focus modeling by mentals on mss enriching known ones.

5.3.2.The branch of theory of algorithms, synthesis of algorithms, where assuming a priory certain classifiers of mdoers are already given algorithms of synthesis of equal constructive versions of those mdoers are developed.

In deductive modes of synthesis those mdoers can include certain axioms and logical statements or can be determined recursively [31]. In the inductive modes, including machine learning, those mdoers can be represented by samples of their domains or their representations, performances of mdoers, others [39].

Certain mss provide methods of transmission, teaching of mss inside of communities $\mathrm{C}$ as well as methods of acquisition of those mss.

While commonality of thesauruses of members of $\mathrm{C}$ let them avoid specification of those methods it becomes unenviable in transmitting and acquiring human mss by computers.

In contrast with machine learning where teachers are forced to provide computers the representations of mss step by step, by portions, teachers can do that holistically and completely when they teach them. The questions arise to the abilities of computers in accepting, disposing and properly processing those mss.

Prospective answers to above questions for RGT problems are presented in [33]-[36], [39][43].

5.3.3. In what follows we analyze how mental ability to communications can be regularized to address then to constructive adequate modeling.

Preliminarily, analyze the above questions for explaining and understanding constituents of communications. 


\section{Regularizing Explaining and Understanding}

6.1. Explanations of mss and their understanding are inseparable constituents of communications in communities.CommunitiesC are unions of people aroused for enhancing effectiveness of resolution of communal problems of members of $\mathrm{C}$ by coordination and cooperation of their efforts.

For those aims people communicate explaining to each other their goals and plans to attain those goals assuming that addressees understand explanations properly, i.e., that original mss and ones activated in addressees are equal to each other.

The premises of proper understanding of each other in $\mathrm{C}$ are the commonalities of their roots and goals evolutionary originated from the roots as well as mssthat people acquire from $C$ to gain memberships of communities Cwhile acquire them together with communicatives ( $\mathrm{cms}$ ) of those mss represented by IDs of mss and, in addition, by samples of indoms of mss if they are do classifiers.

In general, explanations of mss of members of $\mathrm{C}$ are, we assume, representations by, say, resolution, projection or embodiment of cms of those mss aimed to recall, activate, ideally, equal mss of members of $\mathrm{C}$ to cooperate with them in solving common problems.And members of $\mathrm{C}$ understand explanations of mss $\mathrm{m}$ of members of $\mathrm{C}$ if, ideally, activate equal to $\mathrm{m}$ mssm'.

6.2. Focusing cms of type of IDs and assuming that mentals are modeling mssadequately the explaining/understanding of mentals can be specified as follows.

Namely, certain algorithms, mental explainers, correspond to the target mentals $\mathrm{m}$ certain expressions mexp unanimously representing $\mathrm{m}$ while to understand mexpcertain algorithms, mental interpreters, of members of $\mathrm{C}$ correspond to mexp equal to $\mathrm{m}$ mss $\mathrm{m}$ '.

Recalling, that mentals can be interpreted as subnets of colored oriented nets, or graphs, and recalling that those graphs can be isomorphically represented by their matrices of incidents [32] we can state that, ideally, certain explainers correspond to the mentals modeling $\mathrm{m}$ certain expressions comprised from IDs of those mentals, particularly, in the forms of their matrices of incidents, or equal to matrices other expressions of $\mathrm{m}$, while certain interpreters correspond to those expressions certain mentals m' equal to $\mathrm{m}$.

6.3.1. The above explaining /understanding address to the ideal ontological mssof communities, say, mss of Math or programming languages.

Apparently, real communications only approximate the ideal ones.

6.3.2. Referring to explanations of mentals $\mathrm{m}$ by matrices of incidences we emphasize, first of all, the existence of means of transition from $m$ to the equal $m$ '. In languages those matrices are presented by a range of equal them realities, for example, by triples b rels b resolving mentals by rels and incidental to rels nodes b, b which, in fact, can be interpreted as explanations of mss, say, in English, by clauses or their compositions.

6.3.3. Explanations by resolutions can vary in lengths and details depended on the addressee of explanations and can provide additional values by, for example, chaining the clauses consistent with cause-effect or logical inference rules.

6.4. Resuming the above we state that algorithms can adequately specify explaining/understanding of adequate to mssmentalsfor a range of modes used in human communications.

\section{Regularizing Human Computer Communications (HCC)}

7.1. People of communities $\mathrm{C}$ communicate in languages $\mathrm{L}$ of $\mathrm{C}$, and premises for successful communications include, we assume, the following ones: 
- commonality of thesauruses mTh of mss in C, i.e., for the members of C

- commonality of the totalities of cms of mss of mTh, corpuses crpL of L, in C

- rules, syntacies, of correspondences to mss of mThcertain cms-expressions of crpL

-mdoers/algorithms, mental explainers, corresponding cms-expressions to mss

-rules, semantics, corresponding mss to cms-expressions

-mdoers/algorithms, mental interpreters, corresponding mss to cms-expressions.

7.2.1. HCC communications in $\mathrm{C}$ to be comparable with ones between humans, apparently, have to satisfy the above premises.

At present, however ,NCC are based on programming languages ( PL) that meet those premises only partly.

Namely, computers by algorithms, abstract classes and their packages are adequately modeling only mdoers of thesauruses of $\mathrm{C}$.

Programmers assembling those mdoers or their compositions can explain to computers the plans of processing of mdoers that are unanimously understood and realized by computers.

In addition, HCC can be consistent with $\mathrm{C}$ communications wrt systems equally representable by numeric ones as it was illustrated for chess, and therefore [40], allowing to do the same for the adjacent to chess RGT problems.

In the frame of those numerically equal systems, say,the RGT class, inconsistency in HCC wrt pure human communications are inevitable, especially for cms representing emotions, motivations, social rels, etc.

7.2.2. Resuming the above, we assume, that mentals based HCC can approximate C communications to the extent to which constructive cesTh thesauruses of computers are equal to mTh of C.

7.3.1.Let's address now to the requirements to HCCinterpreters of languages L into languages L' in C.

People interpret expressions exsL in L into exsL' in L' of the given languages $\mathrm{L}$ and $\mathrm{L}$ ' of $\mathrm{C}$ by human interpreters Intrs.

Intrs understand exsL, i.e., activate mss $\mathrm{m}^{*}$ equal to ones $\mathrm{m}$ expressed by exsL, then select, pick out exsL' activating mss $m$ ', ideally, equal to $\mathrm{m}^{*}$ for, finally, outputting exsL' expressing m', equal to $\mathrm{m}$.

Apparently, HCC-based interpreters, cIntrs, , ideally, have to perform equally with respect to Intrs.

7.3.2.1.Let's see now to what extent one of the popular UNL cInts [20, 21] meets the above requirements.

UNL originates from the assumptions that thesauruses mThC of communities $\mathrm{C}$ communicating in languages $\mathrm{L}$ and L' converge to communal thesaurus $\mathrm{mTh}$ due to the consequences of the globalization.

As a result, the communities $\mathrm{C}$ and $\mathrm{C}$, it is assumed, do with the same realities and mss representing realities while named by different cms.

Particularly, basic rels of L and L' become equal to some very limited bRels (UNL indicates about 44 of them) that acceptably approximate rels of $\mathrm{L}$ and $\mathrm{L}$ '.

Following those assumptions corresponding to each other mss of C,C' can get the same codes, Universal Words (UW), while any exsL by human Intrs of $\mathrm{C}$ using $\mathrm{UW}$ and bRels can be represented by equal to excL certain UNL expressions exs* that, ideally, would be decoded into equal to exsL expressions exsL' of L'.

7.3.2.2.Unfortunately, there stays unknown any properly acceptable UNL based HCC interpreters during

about 20 years of attempts of realization of UNL ideas in about of 20 languages /countries.

Even it is not proven yet that exsL coded by exs* in UNL by human Ints after some algorithmic decoding into expressions of the same $\mathrm{L}$ will be equal to the original exsL. 
7.3.2.3. Thus, it is natural to revise UNLassumptions.

First, the most doubtful of them, seemingly, concerns the universal basic relsbRels of UNL. Particularly, several researchers have been proving incompleteness of bRels.

Second, the units of languages, particularly, UW only partly representmss while for proper expression of certain mss $m$ the interpreters can be forced to look iteratively for compositions of units of corpuses of languages that will acceptably activate $\mathrm{m}$.

\section{Conclusions}

8.1. We continue studying ontological, constructive and systemic models of mental systems, mss, comparable by expressiveness with algorithms and natural languages.

First, we present the modified specification of the models of mss, mentals, introduced in [47], refine systemic classifiers and constructive regularization of classifiers.

Then, question the ways of proving that mentals can be adequate constructive models of mss to provide arguments of their adequacy for explaining, understanding and human-computer interactions as well as convince to follow the ideas of inventors of algorithms in adequate modeling of mental behavior.

To consist functional and connectivity mental models we provide certain evidence that mentals can be reduced to systems of classifiers, which let us concentrate on the organizing of neurons to link mental doings of neuron nets with mentals.

8.2. Let's remind some still open questions.

Can the patterns of personality of humans including entire ranges of human emotions, motivations and social relationships be equally represented numerically or by mentals?

Can we proceed to a non- numerical representation of relationships, particularly, by neuron nets?

Can we provide certain relationships universal for classes of languages or to prove that it is impossible?

Can we advance in Human-Computer Communications to the extent acceptable, at least, for chess players recalling that chess languages represent the natural ones as drops of water in the oceans? [35].

Can we organize neurons into the nets having mental doings equal to ones of mentals?

\section{References}

[1] R. Feynman, The Meaning of it All, Addison Wesley, Massachusetts, 1998.

[2] J. von Neuman, Theory of Self-reproducing Automata, University of Illinois Press, 1966.

[3] T. Winograd and F. Flores, Understanding Computers and Cognition. A new foundation for design Publishers, Huntington, NY, 1986.

[4] J. Flavell, The Developmental Psychology of Jean Piaget, D.VanNostrand Comp. Inc., Princeton, N.J., 1962.

[5] Z. Pylyshyn, "Seeing and Visualizing: It's Not What You Think”, An Essay on Vision and Visual Imagination, http://ruccs.rutgers.edu/faculty/pylyshyn.html, 2004.

[6] E. Shrodinger, Mind and Matter, Cambridge, 1956.

[7] J. Mandler, The Foundations of Mind: Origins of Conceptual Thought, Oxford Univ. Press, 2004.

[8] A.Turing, Computing Machinery and Intelligence, Mind 49, 1950, [Reprinted in Minds and machines. A. Anderson (ed.), Engelwood Cliffs NJ, Prentice Hall, 1964. 
[9] N. Chomsky, Aspects of the Theory of Syntax, MIT Press, 1965.

[10] J. Pitrat, "Consciousness and conscience, in artificial beings: the conscience of a conscious machine”, ISTE, London, UK, 2009.

[11] K Fu, Syntactic Methods in Pattern Recognition, London, 1974.

[12] E. Zermelo, "Ubereineanwendung der mengenlehre auf die theorie des Shachspiels", Proceedings of the fifth International Conference of Mathematicians, Cambridge University Press, 1912, pp. 501-504.

[13] J. Searle, “Is the brain’s mind a computer program?,Scientific American, vol. 262, pp. 26-31, 1990.

[14] D. Roy, "Grounding language in the world: signs, schemas, and meaning cognitive machines group”, The Media Laboratory, MIT http://www.media.mit.edu/cogmac / projects. html, pp. 1-27, 2005.

[15] C. Shannon, "Programming a computer for playing chess", Philosophical Magazine Ser.7, vol. 41, 1950.

[16] Ch. Brutyan, I. Zaslavski, and L. Mkrtchyan, "On methods of automated synthesis of positional strategies in games”, ProblemiKibernetiki, Moscow, vol. 19, pp. 41-75, 1967.

[17] M. Botvinnik, “About solving approximate problems”, S. Radio, Moscow, (in Russian), 1979.

[18] M. Botvinnik, "Computers in chess: solving inexact search problems”, Springer Series in Symbolic Computation, with Appendixes, Springer-Verlag:, New York, 1984.

[19] A. Elo, The Rating of Chess Players, Past and Present, London, 1978.

[20] J. Laird,The Soar Cognitive Architecture, MIT Press, England, 2012.

[21] (2013) [Online]. Available: UNDL Foundation, www.unlweb.net.

[22] “UNL Specifications”, UNL Center of UNDL Foundation, 2005.

[23] F. Gobet, "Chunking mechanisms in human learning”, Trends in Cognitive Sciences, v.5, pp. 236-243, 2001.

[24] G. Atkinson, Chess and Machine Intuition, Ablex Publishing Corporation, New Jersey, 1993.

[25] J. Furnkranz, "Machine Learning in Games: A Survey in "Machines that Learn to Play Games”, N.Sci., 2001.

[26] J. Moon, Topics on Tournaments, N.Y., Holt, and Winston, 1968.

[27] R. Benergi, Theory of Problem Solving,Mir, Moscow, 1972.

[28] R. Penrose, The Emperor's New Mind, Oxford University Press, 1999.

[29] A. Markov and N. Nagorni, Theory of Algorifms, Nauka, Moscow, 1984.

[30] A. Maltzev, Algorithms and Recursive Functions, Nauka, Moscow, 1965.

[31] H. Marandjian, "A method of synthesis of programs of numeric functions", Mathematical Problems of Cybernetics and Computer, Yerevan,vol. 26, pp. 5-13, 1986.

[32] F. Harary, Graph Theory, Addison-Wesley, 1969.

[33] Perlov D. VilenkinA.Cosmology for the Curious. Springer, 2017

[34] Harari Y.N. Homo Deus: A Brief History of Tomorrow. Harper-Collins, 2017.

[35] Helena Blavatsky: Between Light and Darkness. Жизнь замечательных людей (inRussian). Moscow: Молодаягвардия, 2010.

[36] E.Pogossian and A. Martirosian. "Learning”. Reference book on Intellectual Systems. Radio iSvjas Publishing Company, Moscow, (in Russian), vol. 2, pp. 206-231, 1990.

[37] E. Pogossian, “On modeling cognition”, Computer Science and Information Technologies (CSIT11), Yerevan, 2011, pp 194-198.

[38] E. Pogossian, "Specifying personalized expertise. International Association for Development of the Information Society (IADIS)”, International Conference 
Cognition and Exploratory Learning in Digital Age(CELDA 2006), Barcelona, Spain, pp. 151-159, 2006.

[39] E. Pogossian, V. Vahradyan, and A. Grigoryan, "On competing agents consistent with expert knowledge", Lecture Notes in Computer Science, AIS-ADM-07: The International Workshop on Autonomous Intelligent Systems - Agents and Data Mining, St. Petersburg, Russia, June 6-7, pp. 229-241, 2007.

[40] E. Pogossian, "On measures of performance of functions of human mind", 6th International Conference in Computer Science and Information Technologies, CSIT2007, Yerevan, pp. 149-154, 2007.

[41] E. Pogossian, "On a transparent presentation of written English syntax", 5th Intern. Cognitive Linguistics Conference, VrijeUniversiteit, Amsterdam, July, pp. 209-214, 1996.

[42] E. Pogossian, “Adaptation of combinatorial algorithms”, Academy of Sci. of Armenia, p. 293, Yerevan, 1983.

[43] E. Pogossian, "Effectiveness enhancing knowledge based strategies for SSRGT class of defense problems”, NATO ASI 2011 Prediction and Recognition of Piracy Efforts Using Collaborative Human-Centric Information Systems, Salamanca, Spain, 2011, pp. 16.

[44] Z. Naghashyan and E. Pogossian, "Developing Java software for representation, acquisition and management of strategy knowledge", Mathematical Problems of Computer Sciences, Proc. of IIAP, Yerevan, pp.187-195, 2010.

[45] K. Khachatryan and S. Grigoryan, "Java programs for matching situations to the meanings of SSRGT games”, Proceedings of SEUA Annual conference, Yerevan, Armenia, pp. 135-141, 2013.

[46] S. Grigoryan "Research and Development of Algorithms and Programs of Knowledge Acquisition and Their Effective Application to Resistance Problems”, $P h D, p$ 111, Yerevan, Armenia, 2016.

[47] E. Pogossian, “Towards Adequate Constructive Models of Mental Systems”, 12th International Conference in Computer Science and Information Technologies, CSIT2017, Yerevan, pp. 96-101,2017, 2017, as well as IEEE's Xplore electronic library and is available at the link :http://ieeexplore.ieee.org/xpl/mostRecentIssue.jsp?punumber=8307363

[48] E. Pogossian, S.Grigoryan, N.Hakobyan, "On Systemic Classification and Machine Learning”, 12th International Conference in Computer Science and Information Technologies, CSIT2017, Yerevan, pp.102-107, 2017.

[49] E. Pogossian, “On the Way to Dominating Cognition”, Mathematical Problems of Computer Sciences, Proc. of IIAP, Yerevan, pp.79-91,2018.

[50] S.Grigoryan, N.Hakobyan, H.Vrtanesyan "Object -oriented Modeling of Systemic Classifiers and Matching to Classifiers”, Transactions of IIAP NAS RA, Mathematical Problems of Computer Sciences, Proc. of IIAP, Yerevan, pp. 102-107, 2018.

[51] E. Pogossian, A.Javadyan and E. Ivanyan., "Effective Discovery of Intrusion Protection Strategies.," The International Workshop on Agents and Data Mining, Lecture Notes in Computer Science, St. Petersburg, Russia, pp. 263-274, 2005.

[52] V. Germeier, Games with the Nature, (in Russian), Moscow, Phis-math publishing, 1980.

Submitted 22.08.2018, accepted 02.12.2018. 


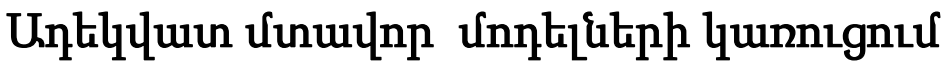

\author{
Ł. Tnпnujuid
}

\section{Uựhnนhnıu}

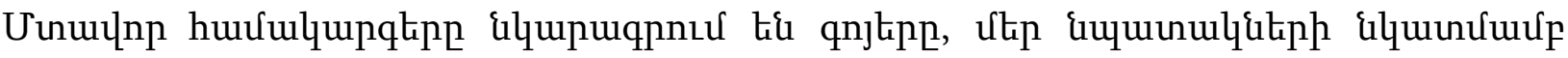

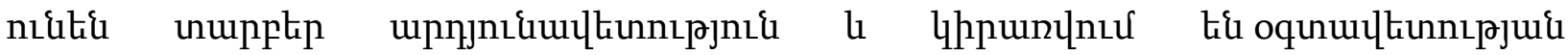
quuuunulqutennu:

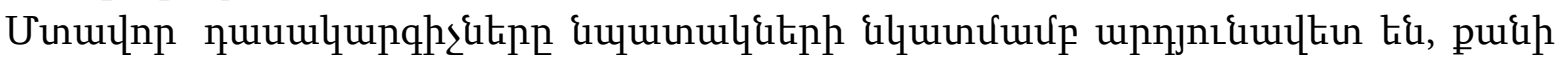

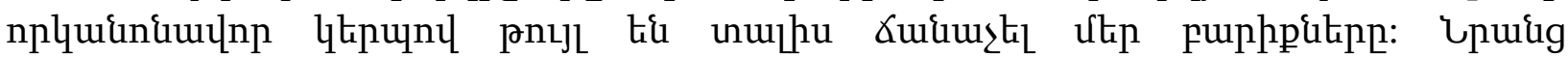

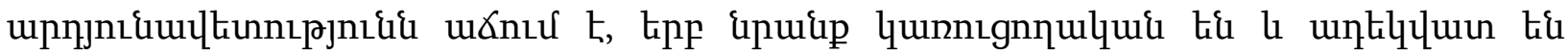
atpluujuginud hpurquinıpjnı\{n:

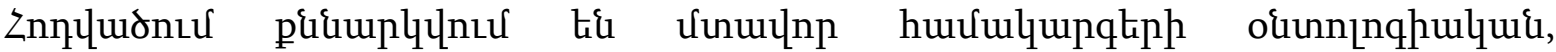

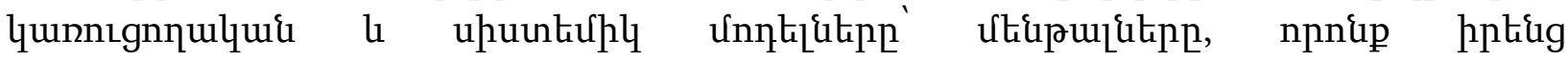

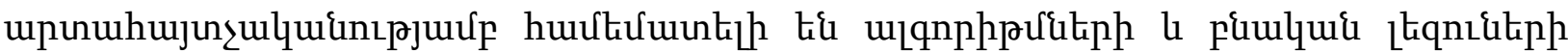

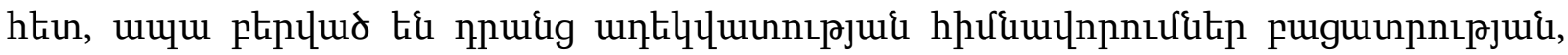

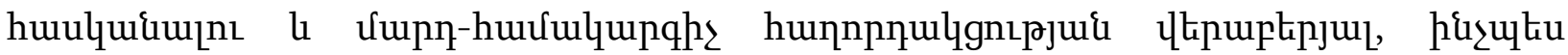

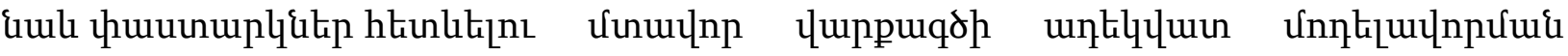

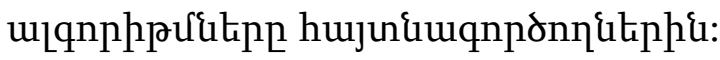

\section{К построению адекватных мыслительных моделей}

\section{Э. Погосян}

\section{Аннотация}

Исследуются онтологические конструктивные модели когнитивных систем, mentals, сравнимые по выразительности с алгоритмами и естественными языками.

В частности, приведены модели обучения, объяснения и понимания при коммуникациях человек-компьютер, а также методы аргументации их адекватности.

Методология построения адекватных моделей исходит из идей конструктивизации вычислимых функций алгоритмами и аргументов обоснования адекватности алгоритмов.

Исходя из сводимости mentals к системам классификаторов уточняются проблемы сближения функциональных/ mentals/ и коннективистских /нейронные сети/ ментальных моделей. 